\title{
Research Paper: Effects of Comprehensive Risk Management Program on the Preparedness of Rofeide Rehabilitation Hospital in Disasters and Incidents
}

\author{
Samira Rajabi ${ }^{1}$, Hamid Reza Khankeh ${ }^{2,3^{*}}$, Vahid Delshad ${ }^{2}$, Mehdi Rahgozar ${ }^{4}$, Narges Arsalani ${ }^{1}$, Farahnaz Mohammadi
}

1. Department of Nursing, University of Social Welfare and Rehabilitation Sciences, Tehran, Iran.

2. Department of Health in Disasters \& Emergencies, University of Social Welfare and Rehabilitation Sciences, Tehran, Iran.

3. Department of Clinical Science and Education, Karolinska Institute, Stockholm, Sweden.

4. Department of Biostatistics, University of Social Welfare and Rehabilitation Sciences, Tehran, Iran.

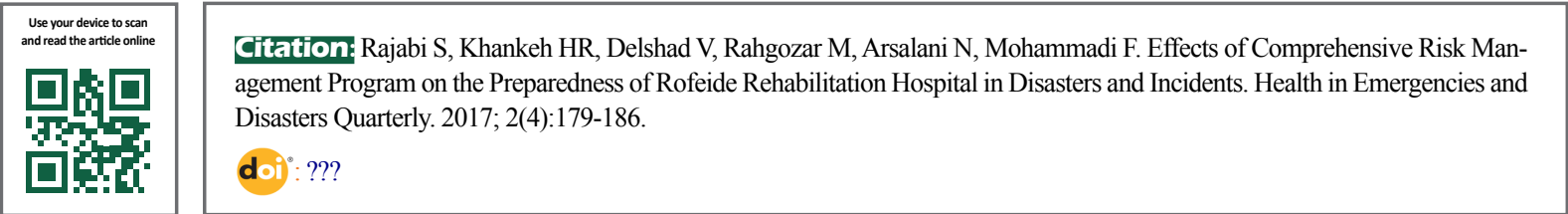

Article info:

Received: 16 Dec. 2016

Accepted: 05 Mar. 2017
Keywords:

Hospital preparedness, Hospital risk management, Disaster and incidents

\section{ABSTRACT}

Background: Iran is exposed to a wide range of natural hazards. The occurrence of unexpected disasters and incidents is mostly destructive and devastating. In such circumstances, the community's ability to meet healthcare needs and demands experiences great challenge. Hospitals are the primary healthcare providers in the communities, and their preparedness is crucial in saving lives during a disaster. Given the importance of hospital risk management program, in this study, we examined the effect of comprehensive risk management on the preparedness of Rofeide Rehabilitation Hospital.

Materials and Methods: This is a pretest-posttest quasi-experimental study. The data were assessed by National Standard Tool through nine components. Before the intervention, the hospital preparedness was assessed using the standard tool through interviews and observations and also completed with the cooperation of Hospital Risk Management Committee. The intervention was designed in two parts including holding a two-day workshop on hospital preparedness for senior managers of hospital and nursing managers. In the next step, the risk assessment, preparation of response plan, and establishment of incident command system were conducted. At the end, the hospital preparedness was compared linearly before and after the intervention.

Results: The hospital preparedness increased from poor to a moderate level by 48 points. Six out of 9 hospital preparedness components including command and control, communications, increased capacity, the continuation of vital services, procurement and logistics, and recovery after disasters were found to be improved after the intervention, and only three components including safety and security, triage, and staffing did not significantly improve.

Conclusion: Considering the positive impact of the implementation of the risk management program on the preparedness of Rofeide Rehabilitation Hospital and promotion of its preparedness level from poor to moderate, as well as relatively high vulnerability of hospitals against internal and external risks, national hospitals are recommended to use the comprehensive hospital risk management model to be more prepared for disasters.

\section{* Corresponding Author:}

Hamid Reza Khankeh, PhD

Address: Department of Health in Disasters \& Emergencies, University of Social Welfare and Rehabilitation Sciences, Tehran, Iran.

E-mail: Hamid.khankeh@ki.se 


\section{Introduction}

\section{$\mathrm{T}$}

he history of human life has always experienced the rush of disasters and incidents. The inevitability of disasters as well as environmental and population interferences of communities has increasingly made all societies vulnerable and also imposed irrecoverable damages on the society, economy, and health of communities worldwide [1].

According to the statistics, there is an increasing incidence of natural disasters worldwide [2]. According to initial statistics of Emergency Events Database (EMDAT) in 2016, the number of disasters across the world has reached to a number of 301 with 102 countries being affected. This is followed by adverse impact on 411 million people and has caused 7628 fatalities and an economic loss of 97 billion US Dollars [3]. In the meantime, Asia has the greatest share of natural disasters among other continents. In 2015, 152 of a total of 346 disasters occurred in Asia [4]. Iran's geographical position in Asia is such that over $90 \%$ of the population is exposed to various disasters and Iran is among the world's top 10 disaster-prone countries $[5,6]$. Given that life, health, and public health have always been affected by disasters, providing healthcare services has helped to restore normalcy in human society after the disasters $[1,6]$. In this respect, the effectiveness of healthcare services in disaster-prone countries such as Iran has been a remarkable issue for those involved in the healthcare system [7].

Hospitals and healthcare centers are the first institutions for post-disaster response [8]. Medical care in hospitals is expected to continue after the disasters, but they would be in trouble to organize the services due to the unpreparedness of the hospitals under such circumstances that results in functional collapse [9]. Dealing effectively with disasters requires implementing a set of activities and designing procedures before and after the incidents, called as hospital risk management measures. Mitigation and preparedness are the pre-disaster measures, whereas response and reconstruction-rehabilitation are among the post-disaster measures [10].

Preparedness as one of the important stages of the risk management cycle leads to an effective response $[6,11]$. As the name implies, this phase predicts the effective measures, plans, activities, and procedures to respond to disasters [11]. Nowadays, preparedness for the prevention of adverse consequences of natural disasters is an international vision and the key to management planning in disaster-prone countries [1]. Studies in the field of hospital preparedness assessment in Iran and other countries indicate their poor preparedness in the face of potential disasters [12-14]. Review studies regarding the post-disaster measures in Iran reflect impairment in the process of treatment [15-17]. Thus, it is very important to maintain preparedness and design plans to respond to incidents and disasters in hospitals. The disaster planning should be a continuous process, and its continuity is of paramount importance [18].

Several studies have been conducted to examine the effect of risk management on the hospital preparedness in Iran, but each of these studies has only assessed a part of the comprehensive risk management on hospital preparedness. For example, Karimiyan et al. examined the impact of training hospital preparedness principles on the preparedness of Motahari Hospital in Tehran [19]. Ghasemi et al. examined the effect of implementation and activation of early warning system on the preparedness of Imam Hospital in Sari [14]. Salavati et al. investigated the impact of training and development of functional and non-structural hospital safety principles on the preparedness of Razi and Dey Hospitals based on the hospital safety index [20]. Thus, it is obvious that previous studies have investigated only one risk management element; therefore, in this study, we examined three primary elements including risk assessment, response plan, and incident command system. To the best of our knowledge, this is the first study in Iran that examines the effect of a more complete package of comprehensive risk management program on the hospital preparedness. It is noteworthy that the aforementioned studies had been conducted in general and specialized hospitals, whereas this study is conducted in a Rehabilitation Hospital.

\section{Materials and Methods}

This is a pretest-posttest quasi-experimental interventional research. The research environment was Rofeide Rehabilitation Hospital affiliated to the University of Social Welfare and Rehabilitation Sciences, Tehran, for the ease of access to information. Rofeide Rehabilitation Hospital is the first scientific and general center to provide various kinds of treatment and rehabilitation services, which was founded in the year 2004 and has 100 approved beds, 5 hospitalization wards, and about 80 active beds. This hospital with its specialized rehabilitation team in various specialties is serving people with disability for the first time in the country at the sub-acute stage.

The intervention in this study has two parts: the first part was a two-day workshop entitled "Hospital Preparedness in Disasters and Incidents" and the second part includes 
following-up the researcher's consultation and teamwork to prepare a list oftop10hospital risks and a response plan and to establish an incident command system.

After receiving the Code of Ethics in Research, the university presented a cover letter to the hospital and the hospital chairman agreed with the study. A list of target group members including senior managers of hospitals and nursing managers were prepared for census, and they were invited to take part in workshops; their consent was also obtained to participate in research. It should be noted that the documentation was completed the day before the workshop of hospital preparedness assessment tool in collaboration with the hospital risk management committee members and through observation and review. The hospital preparedness assessment tool is the national standard tool recommended and notified by the Ministry of Health, Tehran. The general items included in this tool are flexible and extendable for various disasters, which is based on nine important components. Each of these components contains a list of practical and executive necessities for assistance to the authorities at incidents that includes a total of 91 activities.

These nine components are as follows: 1) Command and control (6 activities): Effective Disaster Management depends on the desired performance of the command system. 2) Communications (9 activities): Determination for decision-making, effective participation of employees, transfer of accurate information to the media, and the public require transparent communication. 3) Safety and Security (11 activities): Hospital security and application of security mechanisms according to various risks are essential for the positive performance and response of the hospital. 4) Triage (10 activities): In the face of numerous victims, triage and prioritization of treatment measures are the key components in care of patients. 5) Increased capacity (13 activities): Increased demand in disasters requires promoting the current capacity of hospital to respond effectively. 6) Continuation of vital services ( 8 activities): A disaster or incident is not a reason to remove vital services that are available under normal circumstances. So, with the start of response plan in disasters, the access to vital services should be allowed. 7) Manpower (15 activities): Disasters and incidents require more human resources; thus, it is essential to organize manpower to ensure the continuity of services and empowerment. 8) Procurement and logistics (10 activities): Procurement and supply of hospital requirements and delivering them during incidents are a significant issue. So, this issue needs its own planning. 9) Recovery after disasters (9 activities): In parallel with the start of response plan, the recovery should be taken into consideration; because the speed of the recovery program can have a significant impact on reducing the long-term complications.

In the first phase of the study, the following activities were performed: A total of 91 activities surveyed in the tool are measured with three options, namely under review, under implementation, and completed. According to the expert professors, the score of 1 is assigned to the option "under review," 2 to the option "under implementation," and 3 to the option "completed." At the end, general preparedness of the hospital is placed in one of the three levels: poor (93-152), moderate (153-212), and strong (123-273) [21]. Educational materials presented at the workshop were extracted from the book The hospital preparedness in disasters and incidents: National Plan written by Khankeh et al. [1], which focuses on risk analysis, response plan, and incident command system. In the workshop on risk analysis, the elements involved in this area, including vulnerability, risk, and capacity assessment were explained in detail. Another part of the workshop was dedicated to providing disaster response planning process which described it step-by-step. Then, the participants were asked to work together and develop an example of disaster response plan. Finally, details regarding the incident command system, enabling and disabling the command system, and description of its responsibilities were provided as one of the hospital strategies to deal with disasters.

In the second phase of the study, the following activities were performed: A list of top 10 hospital risks and a response plan was developed for the first five risks through numerous meetings with the participants in the workshop. For the incident command system, the system chart was also provided and various responsible sites were identified, and the duties of the individual positions were described in detail and provided to the members. Ultimately, our intervention package was completed after 2 months with the efforts of the researcher and specialized reviews of the experts (advisor and supervisor, professors, and the workshop lecturers). At the end of the intervention, the hospital preparedness assessment tool was recompleted in collaboration with the hospital disaster and incident management committee members. It should be noted that the committee members who contributed to the completion of the tool were the same before and after the intervention. The difference in overall hospital preparedness and the prep aredness of every single component before and after the intervention was linearly compared. Descriptive statistics were used to analyze the data, and then all dimensions of hospital preparedness before and after the intervention were estimated in tables and statistical charts using ocular method. Ocular 
method is one of the qualitative analysis methods that is used when the sample size is equal to the population [22].

\section{Results}

The participants of the hospital preparedness workshop consisted of 31 senior managers of hospital and nursing managers of Rofeide Rehabilitation Hospital. Of them, 14(45.1) were males and 16(54.8) were females. Demographic characteristics of the participants are presented in Table 1. The scores obtained by each component before and after the intervention were separately and generally investigated based on the scoring methods of hospital preparedness tool. After the study intervention, the general level of hospital preparedness improved from poor (112) to moderate level (160) (Figure 1).

Table 2 shows the scores of nine components before and after the intervention. The component "increased capacity" improved with 14 points and the component "command and control" improved with 7 points, whereas the components "safety and security" and "manpower" improved with 2 points which showed least sensitivity. Command and control was found to be improved from weak to strong level of preparedness, whereas communications, increased capacity, continuation of vital services, procurement and logistics, and recovery after disasters improved from poor to moderate level of preparedness. Other components were found to be at poor level of preparedness and their increased score did not promote the level of preparedness.

\section{Discussion}

Given the vital role of continuing hospital services in risk management cycle [21] as well as their key role in

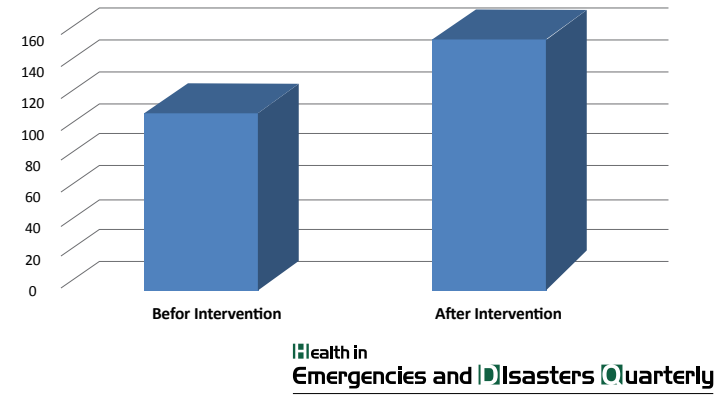

Figure 1. Comparison of hospital preparedness scores before and after the intervention

the risk management cycle and also due to the design of a comprehensive risk management program in the country and to assess the effectiveness of this program, this study aimed to examine the effect of comprehensive risk management program on Rofeide Rehabilitation Hospital preparedness. The study results showed that the hospital preparedness had an obvious improvement in the areas of command and control, communications, increasing capacity, continuity of vital services, procurement and logistics, and recovery after disasters, whereas there was no significant improvement in other areas.

Command and control are of the utmost components in hospital preparedness by which hospitals can respond to the disasters more effectively [22]. The first component in assessing hospital preparedness is command and control, which was in poor status prior to our intervention. Our results are consistent with the results of Yousefi [24] and Ghasemi [14]. However, they were inconsistent with the results of Karimiyan et al. [19] because this component was at a high level of preparedness in Motahari Hospital. The study by Ingrassia et al. also noted the high preparedness level of the command and control in

Table 1. The demographic characteristics of the participants

\begin{tabular}{|c|c|c|}
\hline \multicolumn{2}{|c|}{ Demographic Characteristics } & \multirow{2}{*}{$\begin{array}{c}\text { Number } \\
14\end{array}$} \\
\hline Condor & Male & \\
\hline veriver & Female & 16 \\
\hline \multirow{5}{*}{ Education level } & Associate degree & 3 \\
\hline & BA & 12 \\
\hline & MA & 10 \\
\hline & $\mathrm{PhD}$ & 1 \\
\hline & MD & 5 \\
\hline \multirow{2}{*}{ Experience of relief to victims } & Yes & 6 \\
\hline & No & 25 \\
\hline \multirow{2}{*}{$\begin{array}{l}\text { History of participation in training } \\
\text { courses related to disaster }\end{array}$} & Yes & 16 \\
\hline & No & 15 \\
\hline
\end{tabular}


Table 2. The preparedness scores of nine components before and after the intervention and the preparedness level of tool

\begin{tabular}{|c|c|c|c|}
\hline Preparedness Dimensions & Before the Intervention & After the Intervention & The Score and the Level of Preparedness \\
\hline Command and control & 8 & 15 & Poor: 6-10/Medium: 11-14/strong:15-18 \\
\hline Communications & 12 & 16 & Poor: 9-14 /Medium: 15-20/strong:21-27 \\
\hline Safety and Security & 15 & 17 & Poor: 11-17/Medium:18-25/strong:26-33 \\
\hline Triage & 10 & 14 & Poor:10-17/Medium:18-25/strong:26-33 \\
\hline Increased capacity & 15 & 29 & Poor:13-21/Medium:22-30/strong:31-39 \\
\hline $\begin{array}{l}\text { The continuation of vital } \\
\text { services }\end{array}$ & 10 & 14 & Poor:8-13/Medium:14-19/strong:20-24 \\
\hline Manpower & 17 & 19 & Poor:15-25/Medium:26-36/strong:37-45 \\
\hline Procurement and logistics & 13 & 18 & Poor:10-17/Medium:18-25/strong:26-33 \\
\hline Recovery after disasters & 12 & 18 & Poor:9-14/Medium:15-20/strong:21-27 \\
\hline
\end{tabular}

hospitals of Italy [12]. The lack of job descriptions for the Risk Management Committee members and failure to register and record all documentation relating to the hospital emergency management are among the most important weaknesses of Rofeide Rehabilitation Hospital preparedness.

The initial assessment regarding communications resulted in a poor score, which improved after the intervention which suggests that intervention promoted the hospital's preparedness. The lack of job descriptions for hospital staff about their roles and responsibilities during a disaster, the lack of a mechanism to inform the patient prioritization decisions to staff and institutions, as well as the lack of a suitable method for timely organization and analysis of the information and reporting them to public health officials shows weak communication. One of the most important concerns in Italian hospitals is related to the inadequate communication and preparedness of the hospitals in this area [12]. Yousefi [24] and Delshad [25] also confirm this fact. Zaboli et al. [23] also have assessed the level of communication to be poor which is consistent with our study. Daneshmandi and Hojjat have assessed the preparedness level of communication to be poor and considered the lack of predicting an alternative location for telecommunications and the lack of operating instruc- tions for the communication by staff to the families are among the primary problems [26]. Ingrassia, in his study, implied the importance of a communication plan that includes forecasting backup systems and a reliable way for internal and external information exchange [12].

Increasing capacity component was found to be weak before the intervention, which improved to a moderate level after intervention. Most shortcomings in this area stem from failure to identify the ways of enhancing clinical capacity in the hospital, failure to determine areas for treating the patients over hospital capacity, failure to consider alternative medical locations, failure to identify places near the hospital that can be converted into patient care units, and failure to supply vehicles and alternative plan for the transfer of patients in disasters. These findings were found to be consistent with the results of Yousefi [24] and Delshad [25]. According to Karimiyan [19], increased capacity is at the moderate level. In addition, calculation of the hospital capacity required for the admission of patients and identifying the ways of enhancing the hospital capacity are the strengths of this study.

Continuation of vital services component was found to be weak during the initial assessment, which improved after the intervention. Indexing and prioritizing hospital 
services, determining a plan to maintain the continuity of essential care such as access to air conditioning, and examining the effect of possible events caused by incidents on hospital equipment are among the measures achieved during the study. It should be noted that coordinating with neighboring hospitals and private doctors to continue providing medical services and ensuring the availability of essential items for staying in the hospital are the hospital's strengths. Continuation of vital services component was evaluated to be moderate by Karimiyan [19] and strong by Hosseini Shokouh [27], which is inconsistent with our results. Yousefi [24] and Ghasemi [14] in their research also evaluated this component to be poor, which is consistent with our findings.

Procurement and logistics component was found to be poor during the initial assessment, which improved to a moderate level after the intervention. The issues such as estimating the consumption of essential and medical resources, coordinating with the authorities to ensure the continuous provision of medicines and food supplies for hospitals in the disaster, signing cooperation agreements with vendors to ensure the preparation and delivery of equipment, and other resources in times of scarcity are the most important weaknesses of the hospital. This component was also assessed to be poor by Yousefi [24], Delshad [25], and Ghasemi [14], whereas Hosseini Shokouh [27] assessed more than half of hospitals and found good preparedness status. Karimiyan [19] assessed this component as strong where the strength was storing the items required by the hospital in accordance with the instructions.

Recovery after disaster was also in weak level, and it improved to the moderate level after the intervention. The above finding was consistent with the study of Yousefi [24] and Delshad [25], whereas Karimiyan [19] evaluated this component at moderate level. During the study, failure to appoint an employee to help postdisaster planning and meet the needs of employees and their families as well as inadequacies in determining the time and resources required to complete and repair the hospital in case of evacuation were the evident weaknesses found in this study.

In this study, the components of safety and security, triage, and manpower were at poor level of preparedness. These components did not improve after the intervention despite their increased score. The findings about safety and security and manpower were consistent with the results of Delshad et al. [25]. Zabuli et al. also accounted inadequate staffing organization as the weaknesses of hospital preparedness [23]. According to Yousefi et al. safety and security, triage, and human resources were found to be improved from poor to moderate level [24]. Furthermore, Ghasemi et al. reported high level of safety and security as well as human resources [14].

To explain the lack of improvement in the aforementioned components, it should be said that some recommendations on the necessity of considering safety measures for efficient evacuation of the hospital during disasters; the ways to ensure the accuracy of collecting, storing, and reporting information; and reviewing the reports of hospital safety group were provided in the field of safety and security; however, these recommendations have not been implemented yet. It is noteworthy that a major cause of failure to execute recommended plans is the financial problems. Allocating budget to implement the response program is one of the most important factors to improve the readiness of hospitals and many plans developed in this regard are not implemented due to the lack of providing financial resources [12]. The lack of improvement in the preparedness level of triage in this study can be caused by inactivity of emergency ward at Rofeide Rehabilitation Hospital. It seems that the intersectoral cooperation of affiliated institutions is needed to upgrade hospital preparedness in the field of human resources.

In this study, the initial overall hospital preparedness was found to be poor, which improved after the intervention to a moderate level. According to Yousefi [24] and Ghasemi [14], the hospital preparedness level was poor. In a study conducted to evaluate the hospital preparedness in Italy, 12 out of 15 hospitals showed insufficient preparedness and only three hospitals were effectively prepared [12]. A similar study was conducted in Yemen by Salim Ahmad et al. [13] in which only one hospital had effective preparedness, three hospitals had insufficient, and five hospitals had unacceptable level of preparedness. Among numerous components of disaster management, healthcare centers play an important role. While the findings indicate inadequate preparedness of these centers, the promotion of preparedness in 6 out of 9 components as well as promoting overall hospital preparedness are the strengths of this study. Such studies can play an important role in finding key programs and initiatives that promote hospital preparedness. Accordingly, it is recommended to train the parameters affecting the preparedness and take them seriously in order to observe the progress of our hospitals. One specialty hospital and not having the same hospital for control were the limitations of this study. Therefore, we recommend that a similar hospital should be considered as a control experiment and compare before and after the interven- 
tion in future studies for stronger evidence. This study was conducted in general hospitals; thus, general and specialized hospitals are compared with each other.

\section{Conclusion}

This study contributed to improving six areas: command and control, communications, increased capacity, procurement and logistics, continuity of vital services and recovery after disasters. Thus, it can be said that training, deployment, and implementation of the comprehensive risk management program can improve the hospital preparedness. The healthcare system is recommended to develop a comprehensive risk management program in their hospitals so that managers can implement the program. This way national healthcare centers can operate in a uniform and coordinated manner to increase preparedness against disasters.

\section{Acknowledgments}

Hereby, I appreciate the University of Social Welfare and Rehabilitation Sciences, president, senior managers, and staff of Rofeide Rehabilitation Hospital for assistance in conducting this study, which is a part of my master's thesis.

\section{Conflict of Interest}

The authors declared no conflicts of interest.

\section{References}

[1] Khankeh HR, Co-authors. [Disaster hospital preparedness: National plan (Persian)]. Tehran: University of Social Welfare and Rehabilitation; 2013.

[2] Jassempour K, Shirazi K, Fararooei M, Shams M, Shirazi AR. The impact of educational intervention for providing disaster survival kit: Applying precaution adoption process model. International Journal of Disaster Risk Reduction. 2014; 10: 374-80. doi: 10.1016/j.ijdrr.2014.10.012

[3] EM-DAT. Preliminary data human impact of natural disasters 2016 [Internet]. 2017 [Updated 2017 January 17]. Available From: Http://Www.emdat.be/Publications

[4] CRED. Disasters in Numbers 2015 [Internet]. 2016 [Updated 2016 October 12]. Available From: Http://Www.emdat.be/ Publications.

[5] Ardalan A, Masoomi G, Goya M, Ghaffari M, Miadfar J, Sarvar M, et al. Disaster health management: Iran's progress and challenges. Iranian Journal of Public Health. 2009; 38(1):93-7.
[6] Ghanbari V, Maddah S, Khankeh H, Karimloo M. [The effect of a disaster nursing education program on nurses' disasters preparedness for responding to probable natural (Persian)]. Iran Journal of Nursing. 2011; 24(73):72-80.

[7] Khankeh HR, Khorasani-Zavareh D, Johanson E, Mohammadi R, Ahmadi F, Mohammadi R. Disaster health-related challenges and requirements: A grounded theory study in Iran. Prehospital and Disaster Medicine. 2011; 26(03):151-8. doi: $10.1017 /$ s1049023x11006200

[8] Charney RL, Rebmann T, Esguerra CR, Lai CW, Dalawari P. Public perceptions of hospital responsibilities to those without medical Presenting injury or illness during a disaster. The Journal of Emergency Medicine. 2013; 45(4):578-84. doi: 10.1016/j.jemermed.2013.05.010

[9] Djalali A, Castren M, Khankeh H, Gryth D, Radestad M, Öhlen G, et al. Hospital disaster preparedness as measured by functional capacity: A comparison between Iran and Sweden. Prehospital and Disaster Medicine. 2013; 28(05):454-61. doi: 10.1017/s1049023x13008807

[10] Saldana-Zorrilla SO. Assessment of disaster risk management in Mexico. Disaster Prevention and Management: An International Journal. 2015; 24(2):230-48. doi: 10.1108/dpm11-2013-0201

[11] Esfandiari A, Heidari A, Julaee H, Rahimi S, Salari H. Survey of natural disasters preparedness in public and private hospitals of Islamic Republic of Iran (case study of Shiraz, 2011). International Journal of Health System and Disaster Management. 2013; 1(1):26. doi: 10.4103/2347-9019.122441

[12] Ingrassia PL, Mangini M, Azzaretto M, Ciaramitaro I, Costa L, Burkle FM Jr, et al. Hospital disaster preparedness in Italy: a preliminary study utilizing the World Health Organization hospital emergency response evaluation toolkit. Minerva Anestesiologica. 2016; 82(12):1259-66. PMID: 27270072

[13] Aladhrai SA, Djalali A, Della Corte F, Alsabri M, El-Bakri NK, Ingrassia PL. Impact of the 2011 revolution on Hospital Disaster Preparedness in Yemen. Disaster medicine and public health preparedness. 2015; 9 (04): 396-402.

[14] Qassemi F, Khankeh H, Delshad V, Hosseini M. The effects of implementing early warning system and activating the Imam Khomeini on the preparedness of Sari Hospital (RH) in disasters and incidents. Health in Emergencies and Disasters Quarterly. 2016; 2(1):19-24. doi: 10.18869/nrip.hdq.2.1.19

[15] Khankeh HR, Mohammadi R, Ahmadi F. [Barriers and facilitators of health care services at the time of natural disasters (Persian)]. Archives of Rehabilitation. 2005; 6(1):23-30.

[16] Khankeh HR, Mohammadi R, Ahmadi F, Maddah SB, Ranjbar M, Khodaei MR. [Management of health care services at time of natural disasters (Persian)]. Archives of Rehabilitation. 2006; 7(2):49-55.

[17] Khankeh HR, Mohammadi R, Ahmadi F. [Health care services at time of natural disasters: A qualitative study (Persian)]. Iran Journal of Nursing. 2007; 20(51):85-96.

[18] Bazregar R, Khankeh H, Ahmadi S, Hosseini M, Rahgozar M, Moradian M. [The evaluation of application of coordination model based disaster response in disaster Preparedness Rajaye hospital (Persian)]. Iranian Journal of Nursing Research. 2013; 29(8):10-118. 
[19] Karimiyan A, Khankeh H, Dalvandi A, Farzin Nia B. The effect of teaching principles of hospital preparedness according to the national program on preparedness of Shahid Motahari burns hospital of Tehran in response to disasters. Health in Emergencies and Disasters Quarterly. 2017; 2(1):25-32

[20] Salevaty J, Khankeh H, Dalvandi A, Delshad V. The impact of nurses training and applying functional and nonstructural hospital safety in hospitals in disasters preparedness of razi and day based on hospital safety index. Health in Emergencies and Disasters Quarterly. 2015; 1(1):17-24

[21] Khankeh H, Co-authors. [Risk assessment tools and indicators of surge capacity of health in disasters (Persian)]. Tehran: University of Social Welfare and Rehabilitation; 2016.

[22] Karimian A. The effect of education based on national guideline on hospital disaster preparedness and response Burn martyr Motahari Hospital, Tehran to Disaster [MSc. thesis]. Tehran: University of Social Welfare and Rehabilitation 2014.

[23] Zaboli R, Sajadi HS. Assessing hospital disaster preparedness in Tehran: Lessons learned on disaster and mass casualty management system. International Journal of Health System and Disaster Management. 2014; 2(4):220-4.

[24] Yousefi S. [National hospital preparedness and risk management and safty guidline on hospital staff preparedness assessment tool using simulation and national risk (Persian)] [MSc thesis]. Tehran: University of Social Welfare and Rehabilitation; 2017.

[25] Delshad V, Borhani F, Khankeh H, Abbaszadeh A, Sabzalizadeh S, Moradian MJ, et al. The effect of activating early warning system on motahari hospital preparedness. Health in Emergencies and Disasters. 2015; 1(1):3-8.

[26] Daneshmandi M, Amiri H, Vahedi M, Farshi M, Saghafi A, Zigheymat F. Assessing level of preparedness for confronting the crisis such as flood, earthquake, fire and storm in some selected hospitals of Iran. Iranian Journal of Military Medicine. 2010; 12(3):167-71.

[27] Hosseini Shokouh SM, Arab M, Rahimi A, Rashidian A. Sadr Momtaz N. [Preparedness of the Iran University of Medical Sciences hospitals against earthquake (Persian)]. Journal of School of Public Health and Institute of Public Health Research. 2009; 6(3):61-77. 